\title{
Modulation of Circulating Macrophage Migration Inhibitory Factor in the Elderly
}

\author{
Christos Rammos, Ulrike B. Hendgen-Cotta, Julia Pohl, Matthias Totzeck, Peter Luedike, \\ Volker T. Schulze, Malte Kelm, and Tienush Rassaf
}

Division of Cardiology, Pulmonology and Vascular Medicine, Medical Faculty, University Hospital Düsseldorf, Moorenstraß 5, 40225 Düsseldorf, Germany

Correspondence should be addressed to Tienush Rassaf; tienush.rassaf@med.uni-duesseldorf.de

Received 11 June 2014; Accepted 20 June 2014; Published 8 July 2014

Academic Editor: Elísio Costa

Copyright (c) 2014 Christos Rammos et al. This is an open access article distributed under the Creative Commons Attribution License, which permits unrestricted use, distribution, and reproduction in any medium, provided the original work is properly cited.

\begin{abstract}
Aging increases the risk for cardiovascular morbidity and mortality. Chronic low-grade inflammation deteriorates vascular function, increases age-related vascular stiffness, and affects hemodynamics. The proinflammatory cytokine macrophage migration inhibitory factor (MIF) is a major mediator of atherosclerosis. Plasma MIF levels are associated with arterial stiffness, a hallmark of vascular aging. Preclinical studies show that blockade of MIF leads to atherosclerotic plaque regression. Nutritional approaches provide opportunities to counteract age-related inflammation. Following a chronic dietary supplementation with the micronutrient nitrate has been demonstrated to improve vascular stiffness. Whether dietary nitrate affects circulating MIF levels is not known. In a randomized placebo-controlled, double-blinded study, elderly subjects received a dietary nitrate supplementation for 4 weeks. Dietary nitrate led to a decrease in plasma MIF levels in the elderly and to an improvement in vascular functions. This was associated with a reduction in central systolic blood pressure. Our data show that supplementation with dietary nitrate is associated with a reduction of circulating MIF levels along with an improvement in vascular function. This supports the concept of dietary approaches to modulate age-related changes of vascular functions.
\end{abstract}

\section{Introduction}

The physiological aging process leads to deterioration of vascular integrity and homeostasis and these alterations have a relevant effect on cardiovascular disease [1]. A recent study demonstrated that vascular stiffness in the elderly precedes and contributes to incident hypertension with an increase in cardiovascular morbidity and mortality [2]. Dysfunctional endothelium and increased vascular stiffness are the main features of preclinical atherosclerosis and aging blood vessels supply the environment for vascular disease progression. A potential cause of vascular dysfunction within advancing age is considered the chronic low-grade inflammation of the vessel wall [3]. The upregulation of the inflammatory response is the consequence of a remodeling of the innate and acquired immune system with a chronic inflammatory cytokine production $[4,5]$.

The proinflammatory cytokine macrophage migration inhibitory factor (MIF) has gained attention due to its proatherogenic properties [6]. MIF was shown to be involved in inflammatory atherosclerosis pathogenesis and is implicated in modulation of disease progression $[7,8]$. Clinical evidence indicates an association of MIF plasma levels with diminished endothelial function and increased vascular stiffness in patients with established cardiovascular risk [9]. Plaque regression and a more stable plaque phenotype have been shown through MIF blockade in a preclinical setting [6]. Whether modulation of circulating plasma MIF levels is feasible has not been investigated so far.

Despite efforts in pharmacological advances and dedicated medical treatment options, the cardiovascular disease burden remains high in the elderly population [10]. A healthy lifestyle and in particular a healthy diet may support physiological and healthy aging. Nutritional interventions and appropriate diets provide options to delay or even counteract age-related inflammation [11]. Adherence to certain dietary patterns is of significant importance and has been shown to impact mortality [12]. A short-term dietary 
nitrate supplementation affects vascular function and acutely lowers diastolic blood pressure in young volunteers [13]. The micronutrient inorganic nitrate is abundant in leafy green vegetables and is metabolized in vivo to nitrite, nitric oxide, and other nitrogen oxides [14]. This is particularly relevant, as nitric oxide regulates cardiovascular homeostasis under physiological and pathological conditions [15-17]. In elderly volunteers with moderately increased cardiovascular risk, a chronic dietary nitrate supplementation reversed age-related vascular dysfunction and improved prognostic relevant outcome measures [18].

Based on these findings we sought to investigate the effect of a chronic dietary nitrate supplementation on circulating MIF levels following improved vascular functions.

\section{Methods}

2.1. Study Population. The study was performed in accordance with the Declaration of Helsinki, and the Heinrich-Heine University Dusseldorf Institutional Ethics Committee approved the study protocol (Clinicaltrials.gov NCT01729234). 21 elderly volunteers gave informed consent and were included in a randomized, placebo-controlled double-blind trial, as published previously [18]. One volunteer was excluded in the present analysis due to a gastrointestinal disorder at baseline. Volunteers received dietary nitrate (sodium nitrate $150 \mu \mathrm{mol} / \mathrm{kg}$ body weight; dose is equivalent to a portion of spinach) for 4 weeks and were compared to control (sodium chloride $150 \mu \mathrm{mol} / \mathrm{kg}$ body weight), as previously described [18]. Measurements were performed before and 1 day after the last intake of the 4-week ingestion regimen with nitrate and placebo, respectively.

Blood was drawn for clinical routine and the Institute of Clinical Chemistry and Laboratory Diagnostics, University Hospital Dusseldorf, performed all analyses unless noted otherwise.

2.2. MIF Plasma Levels. MIF was determined as described previously [19]. Briefly, heparinized full blood was centrifuged at $800 \mathrm{~g}$ for $10 \mathrm{~min}$ ( $4^{\circ}$ Celsius). The resulting plasma aliquots were snap-frozen in liquid nitrogen and stored at $-80^{\circ}$ Celsius until further analysis. MIF levels were measured by quantitative sandwich enzyme-linked immunosorbent assay (ELISA) (Quantikine, R\&D Systems, Minneapolis, USA) according to the manufacturer's protocols.

2.3. Central Hemodynamics. Brachial blood pressure (BP) was measured in duplicate by cuff and mercury sphygmomanometer after participants had rested in a seated position for $10 \mathrm{~min}$ and the average of 2 measurements was recorded. The indirect measures of central hemodynamics were obtained with the subject in a supine position by using the SphygmoCor system (AtCor Medical, Sydney, Australia) as previously described [9]. Radial arterial pressure waveforms were obtained by applanation tonometry and central arterial waveforms were generated using a validated inbuilt transfer function. Applanation tonometry has been validated to yield precise assessments of intra-arterial pressures by comparison with simultaneous invasive pressure recordings [20]. The system provided a corresponding central aortic pulse waveform from which central SBP (cSBP), central DBP, and augmentation pressure (AP) are identified.

2.4. Statistical Methods. Results are expressed as mean \pm standard error (SEM). Differences between groups were compared using unpaired Student's two-tailed $t$-test. Within group analysis was conducted with Student's paired $t$-test. Correlations between individual parameters were calculated using univariate analyses. Results are expressed as Pearson's $r$ and corresponding $P$ values. $P$ values of less than 0.05 were regarded statistically significant. All statistical tests were conducted using SPSS 21.0 (IBM) and Prism 5.0 (GraphPad) for Mac OS.

\section{Results and Discussion}

Aging is the key nonmodifiable cardiovascular risk factor and leads unequivocally to diverse disadvantageous alterations of the cardiovascular system with the consequence of increased cardiovascular morbidity and mortality [1]. Strategies to delay the cardiovascular aging process are needed. We included community-dwelling elderly volunteers, representative for the general population. Subjects had an increased cardiovascular risk with moderately increased heart score, elevated cholesterol levels, slightly increased body mass index, and mild systolic hypertension. No history, signs, or symptoms of cardiovascular disease were noted. Baseline characteristics are given in Table 1. The moderate increase in cardiovascular risk is in line with studies examining healthy, wellfunctioning men and women free of cardiovascular disease in the general population $[21,22]$.

A typical feature and possible key mechanism of the vascular aging process is believed to be a chronic, low-grade inflammatory status [3]. Actually, inflammation has emerged as one potential cause in the pathogenesis of major agerelated diseases such as atherosclerosis, type 2 diabetes, and renal diseases [23-25].

3.1. Age-Related MIF Expression. The pleiotropic cytokine MIF has been attributed proinflammatory properties and is involved in atherogenic and in particular cardiovascular disease progression [8, 26, 27]. Recent reports have substantiated the inflammatory link by demonstrating that the MIF expression is linked to NF- $\kappa \mathrm{B}$ signaling network and that proinflammatory stimuli can activate the expression of MIF [28]. Age-related modulation was unknown until now. In the present study we determined increased MIF plasma levels in the elderly compared to young controls (65.5 \pm $4.1 \mathrm{ng} / \mathrm{mL}$ versus $17.2 \pm 0.5 \mathrm{ng} / \mathrm{mL}, P<0.0001$, Figure 1$)$. Recently, we used gene expression microarray technology to determine age-related changes of the vascular transcriptome in mice. Upregulated MIF levels were shown in the old aorta compared to young, which emphasizes an age-related transcriptional regulation of MIF in vascular tissue [29]. 
TABLE 1: Baseline patient characteristics.

\begin{tabular}{lccc}
\hline & Control & Nitrate & \\
& $n=10$ & P value & 0.66 \\
Age (years) & $62.6 \pm 1.3$ & $63.7 \pm 2$ & 0.87 \\
Sex (men/women) & $6 / 4$ & $7 / 4$ & 0.98 \\
Heart score & $4.7 \pm 1$ & $4.7 \pm 1$ & 0.13 \\
BMI $\left(\mathrm{kg} / \mathrm{m}^{2}\right)$ & $26.2 \pm 0.8$ & $23.9 \pm 1.2$ & 0.41 \\
Creatinine $(\mathrm{mg} / \mathrm{dL})$ & $0.8 \pm 0.04$ & $0.9 \pm 0.06$ & 0.32 \\
Hemoglobin $(\mathrm{g} / \mathrm{dL})$ & $14.5 \pm 0.4$ & $14.1 \pm 0.2$ & 0.38 \\
Triglycerides (mg/dL) & $104 \pm 13$ & $126 \pm 21$ & 0.40 \\
Cholesterol (mg/dL) & $219 \pm 14.1$ & $236 \pm 12.2$ & 0.47 \\
HDL (mg/dL) & $68 \pm 6$ & $62 \pm 7$ & 0.91 \\
LDL (mg/dL) & $154 \pm 9$ & $156 \pm 13$ & 1.00 \\
Sodium (mmol/L) & $142 \pm 0.6$ & $142 \pm 0.6$ & 0.34 \\
Potassium (mmol/L) & $4.1 \pm 0.1$ & $4.2 \pm 0.1$ & 0.33 \\
CRP (mg/dL) & $<0.3$ & $<0.3$ & 0.15 \\
Hbalc (\%) & $5.6 \pm 0.1$ & $5.8 \pm 0.1$ & \\
\hline
\end{tabular}

Selected demographic, clinical, and biochemical parameters. Values are expressed as mean \pm SEM.

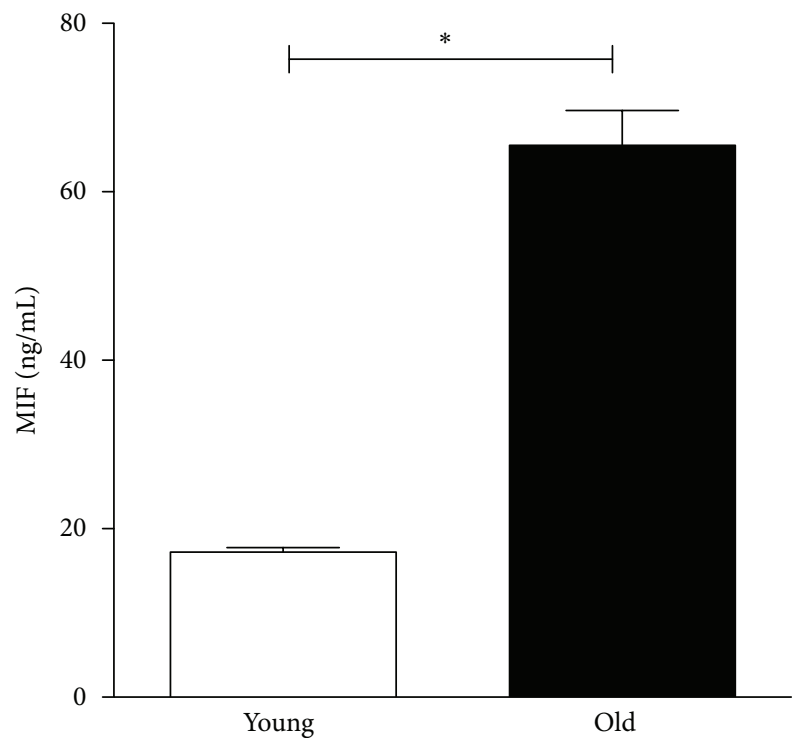

FIGURE 1: MIF plasma levels are increased in old healthy volunteers compared to young controls ( $*$ denotes $P<0.05$ ).

Corroborating MIF's role in the age-related atherogenesis process we further found significant associations with established risk factors. Homocycteine, a recognized cardiovascular risk factor, was related to MIF in the present study $(r=0.64, P=0.002$, Figure 2(a)) [30]. Plasma high-density lipoprotein levels were associated inversely with MIF plasma levels $(r=-0.64, P=0.006)$. The contrariwise relation is in line with the notion that HDL has anti-inflammatory properties [31]. These results are in line with MIF's involvement in the preclinical atherosclerosis process based on low-grade inflammation [26]. Further mechanistic studies have to prove a direct link here. Importantly, experimental data suggest a beneficial effect through MIF blockade with impaired T-cell recruitment and atherosclerotic plaque regression $[6,32]$.

A preclinical study suggested that MIF plays a role in controlling mammalian life span. The authors observed that MIF-deficient mice lived longer than their control counterparts [33]. Thus, targeting MIF with pharmacological and nonpharmacological options seems an important matter to be addressed, with possible beneficial effects affecting health span or even longevity.

3.2. Dietary Nitrate Reduces MIF Levels. Nutritional interventions and lifestyle interventions are the cornerstone of cardiovascular disease prevention strategies. In particular, adherence to a Mediterranean dietary pattern is of great importance and has been shown to impact mortality [12]. Although there exists dispute regarding the effect of definite micro- and macronutrients on vascular functions, the groundbreaking DASH (Dietary Approaches to Stop Hypertension) trial demonstrated that certain dietary patterns influence blood pressure [34-36]. Abundant in our everyday diet and especially in leafy green vegetables is the micronutrient inorganic nitrate. Nitrate can be bioactivated via the reduction to nitrite by symbiotic bacteria in the oral cavity and is consecutively converted to nitric oxide (NO) [3739]. A dietary nitrate supplementation has thus emerged as a possibility to enhance $\mathrm{NO}$ signaling and replenish $\mathrm{NO}$ bioavailability $[14,40]$. Improved vascular remodeling was demonstrated in an animal model of hind-limb ischemia after dietary nitrate intervention [37]. In healthy volunteers ingestion of inorganic nitrate enhanced blood flow in combination with a reduction in blood pressure [41]. We showed that a dietary nitrate supplementation improves endothelial dysfunction and vascular stiffness in the elderly [18]. These outcome measures have been shown to predict cardiovascular events in old adults [42, 43]. Succeeding the reversal of agerelated vascular dysfunction with accompanied increased 


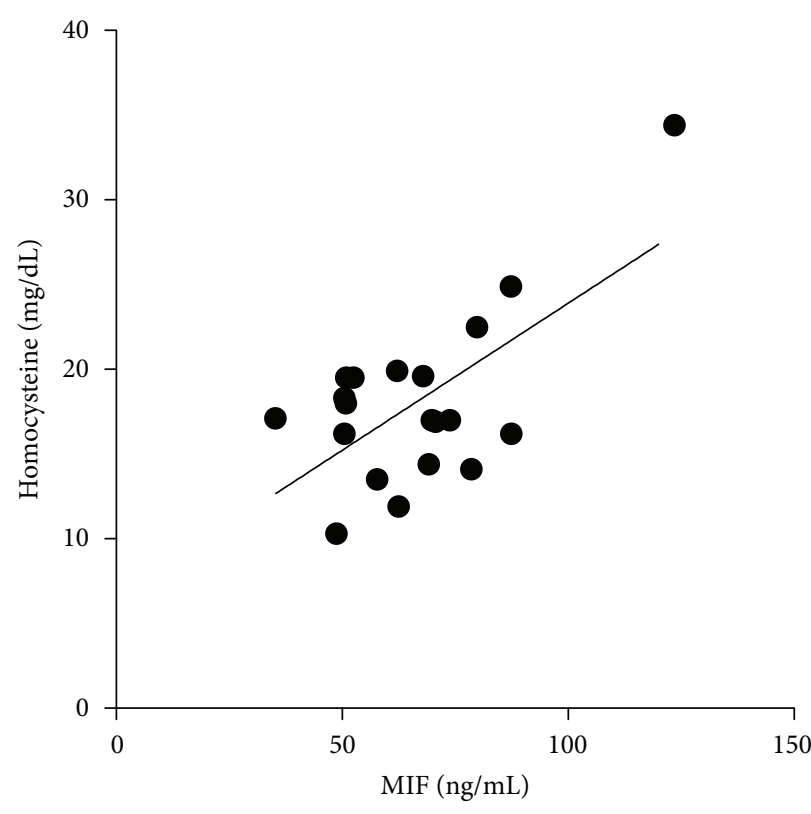

(a)

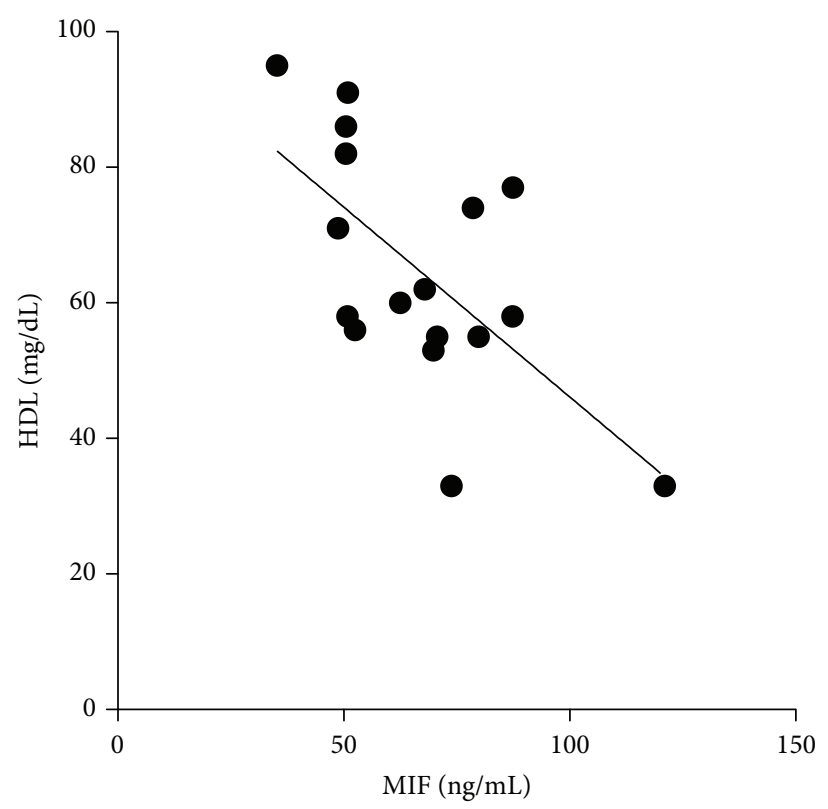

(b)

FIGURE 2: (a) MIF levels are correlated with homocysteine levels in the elderly $(r=0.64, P=0.002)$. (b) MIF plasma levels are related to high-density lipoproteins (HDL, $r=-0.64, P=0.006$ ).

nitrate and nitrate levels [18], we now determined reduced MIF levels following a 4-week dietary nitrate supplementation $(69.8 \pm 7.1 \mathrm{ng} / \mathrm{mL}$ to $49.3 \pm 3.7 \mathrm{ng} / \mathrm{mL}, P<0.05$, Figure 3$)$. No effect was observed for controls $(62.9 \pm 4.8 \mathrm{ng} / \mathrm{mL}$ to 63.3 $\pm 6.0 \mathrm{ng} / \mathrm{mL}, P>0.05$ ). Clearly, this study cannot provide a mechanistic link. It remains elusive whether reduced MIF levels are the consequence of dietary nitrate treatment due to increased NO bioavailability or the result of improved vascular functions. Possible interactions following a nitraterich diet have to be considered. MIF has previously been shown to be targeted by S-nitrosylation and to improve cardioprotection after acute ischemia and reperfusion through modulation of intrinsic oxidoreductase activity $[44,45]$. Whether dietary nitrate additionally modifies MIF's function in the present study remains speculative and should be addressed in further research. Nonetheless, there exist efforts to counteract the age-related low-grade inflammation through dietary approaches [46]. In a preclinical study dietary nitrite supplementaton was shown to modulate agerelated inflammatory cytokines in mice [47]. We, however, only focused on the role of dietary nitrate on MIF. Further studies are necessary to investigate effects on other cytokines.

3.3. Central Hemodynamics. Chronic inflammation directly influences premature atherosclerosis and arterial stiffness [48]. Vascular stiffening is considered one of the most important alterations to aged vessels. It can be measured noninvasively and is regarded as an independent predictor of cardiovascular disease events $[43,49,50]$.

Increased vascular stiffness affects timing and magnitude of central pulse wave reflections, altering central hemodynamics. Consequently ventricular loading conditions and

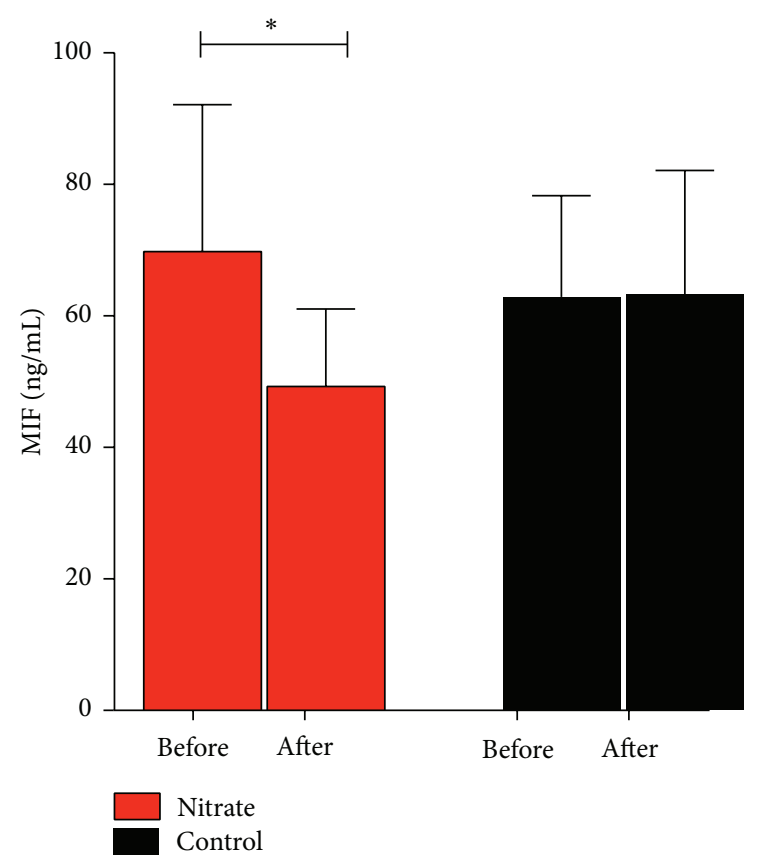

Figure 3: MIF plasma levels are decreased after chronic dietary nitrate supplementation in elderly volunteers $(*$ denotes $P<0.05)$.

coronary blood flow are disturbed and the risk for endorgan damage is elevated [51]. This impacts on cardiac functions, limiting prognosis, and is thus implemented in recent guidelines [52].

Improvements in peripheral vascular functions following nitrate supplementation with reduced proinflammatory MIF 


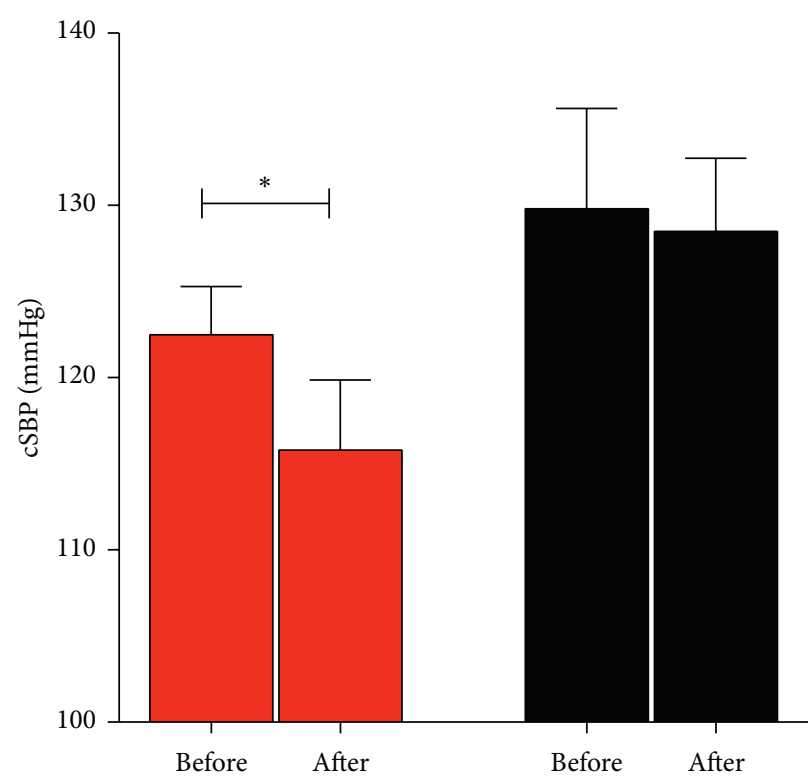

(a)

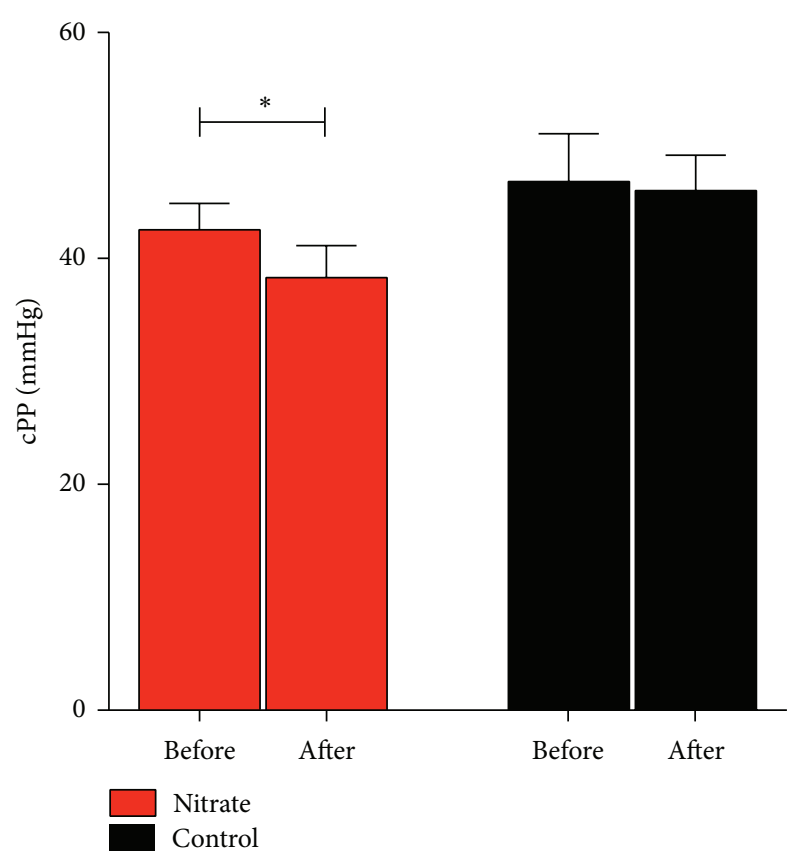

(c)

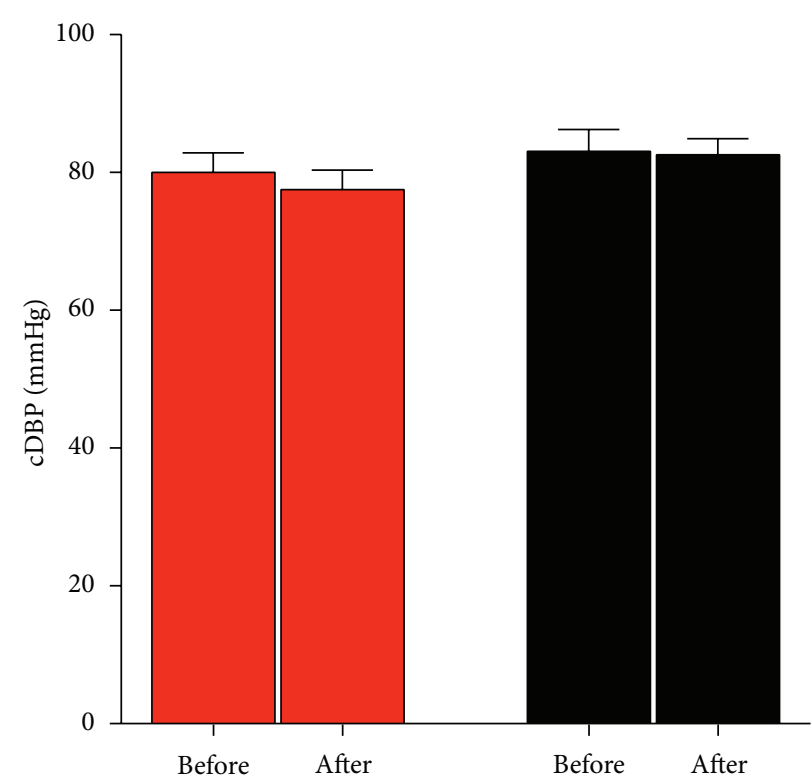

(b)

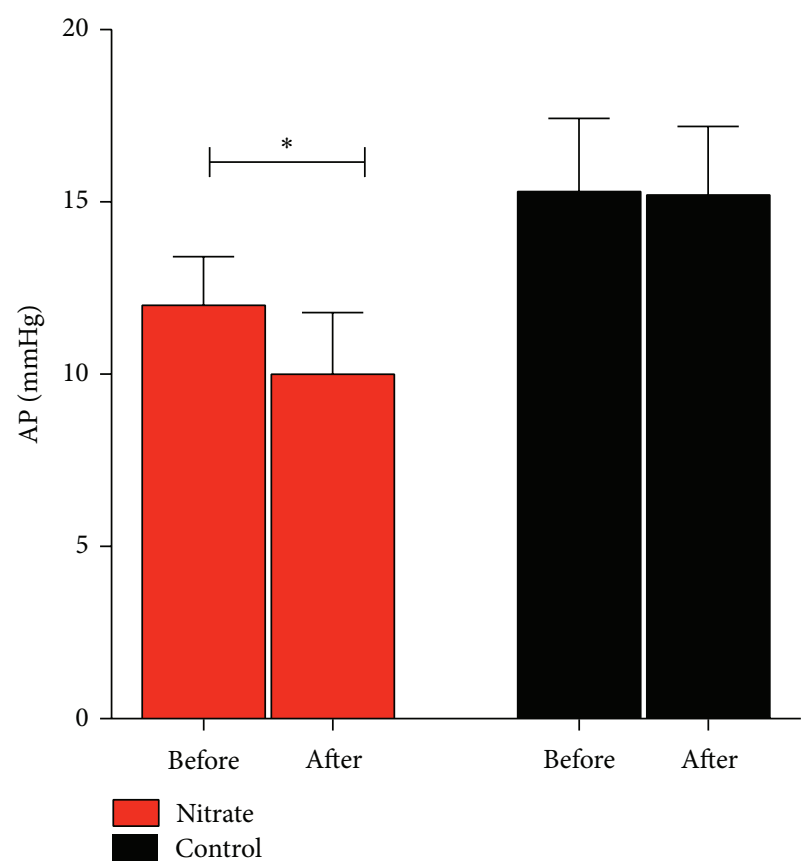

(d)

FIGURE 4: Dietary nitrate improves central hemodynamics and arterial stiffness. (a)-(b) Dietary nitrate reduced central systolic blood pressure (cSBP), while central diastolic blood pressure (cDBP) remains unaffected. (c) Central pulse pressure (cPP) was reduced following nitrate supplementation. (d) Augmentation pressure (AP) was decreased after nitrate rich diet $(* \operatorname{denotes} P<0.05)$.

levels suggest an effect on central hemodynamics. Reduced central systolic blood pressure was determined in the elderly after nitrate supplementation with no effect on controls (nitrate $122.5 \pm 2.8 \mathrm{mmHg}$ to $115.8 \pm 4.0 \mathrm{mmHg}, P<0.05$, and controls $129.8 \pm 5.8 \mathrm{mmHg}$ to $128.5 \pm 4.3 \mathrm{mmHg}, P=$ ns, Figure 4(a)). This was accompanied by reduced central pulse pressure $(\mathrm{cPP}$ : nitrate $42.5 \pm 2.3 \mathrm{mmHg}$ to $38.3 \pm$ $2.8 \mathrm{mmHg}, P<0.05$, and controls $46.8 \pm 4.2 \mathrm{mmHg}$ to
$46.0 \pm 3.2 \mathrm{mmHg}, P=$ ns, Figure 4(b)). No effect was observed for central diastolic blood pressure in nitrate treated elderly volunteers $(P=\mathrm{ns}$, Figure $4(\mathrm{c}))$. Following inorganic nitrate ingestion, reduction in diastolic blood pressure has been described in healthy young volunteers before $[13,53]$. We here, however, investigate elderly subjects, who show alterations in vascular integrity and function. We therefore suggest an age-dependent effect of dietary nitrate, with no 
effect on diastolic blood pressure. Improvement in central blood pressure after nitrate supplementation was substantiated by reduced wave reflection and stiffness indices assessed by augmentation pressure (nitrate $12.4 \pm 1.6 \mathrm{mmHg}$ to $9.4 \pm$ $1.7 \mathrm{mmHg}, P<0.05$, and controls $15.3 \pm 2.1 \mathrm{mmHg}$ to $15.2 \pm$ $2.0 \mathrm{mmHg}, P=\mathrm{ns}$, Figure $4(\mathrm{~d}))$. These results are in line with studies showing attenuation of inflammation through dietary interventions and associated reductions in arterial stiffness [54]. The observed hemodynamic changes may thus implicate reduced cardiovascular risk in the elderly following dietary nitrate supplementation.

\section{Conclusion}

Our findings show that MIF levels are increased in the elderly. Following a chronic dietary nitrate supplementation, reduced MIF plasma levels are observed. Improvement in vascular functions and inflammation is substantiated by improved central hemodynamics in the elderly. This supports the concept of a dietary approach to modulate age-related vascular alterations.

\section{Conflict of Interests}

The authors declare that there is no conflict of interests regarding the publication of this paper.

\section{Acknowledgments}

The authors would like to thank the subjects who participated in this study and A. Bernard and P. Stock for excellent technical help. This study was supported by grants from the German Research Foundation (DFG) to Tienush Rassaf (Ra969/6-1). TR is a Heisenberg professor funded by the DFG (Ra969/7-2). MT and PL received funding from the Forschungskommission Heine-Heine-University, Düsseldorf, Germany.

\section{References}

[1] E. G. Lakatta and D. Levy, "Arterial and cardiac aging: major shareholders in cardiovascular disease enterprises. Part I: aging arteries: a "set up" for vascular disease," Circulation, vol. 107, no. 1, pp. 139-146, 2003.

[2] B. M. Kaess, J. Rong, M. G. Larson et al., "Aortic stiffness, blood pressure progression, and incident hypertension," The Journal of the American Medical Association, vol. 308, no. 9, pp. 875-881, 2012.

[3] C. Franceschi, M. Capri, D. Monti et al., "Inflammaging and anti-inflammaging: a systemic perspective on aging and longevity emerged from studies in humans," Mechanisms of Ageing and Development, vol. 128, no. 1, pp. 92-105, 2007.

[4] D. Baylis, D. B. Bartlett, H. P. Patel, and H. C. Roberts, "Understanding how we age: insights into inflammaging," Longevity \& Healthspan, vol. 2, article 8, 2013.

[5] A. Salminen, J. Huuskonen, J. Ojala, A. Kauppinen, K. Kaarniranta, and T. Suuronen, "Activation of innate immunity system during aging: NF- $\kappa \mathrm{B}$ signaling is the molecular culprit of inflamm-aging," Ageing Research Reviews, vol. 7, no. 2, pp. 83105, 2008.
[6] J. Bernhagen, R. Krohn, H. Lue et al., "MIF is a noncognate ligand of CXC chemokine receptors in inflammatory and atherogenic cell recruitment," Nature Medicine, vol. 13, no. 5, pp. 587-596, 2007.

[7] A. Burger-Kentischer, H. Goebel, R. Seiler et al., "Expression of macrophage migration inhibitory factor in different stages of human atherosclerosis," Circulation, vol. 105, no. 13, pp. 15611566, 2002.

[8] A. Zernecke, J. Bernhagen, and C. Weber, "Macrophage migration inhibitory factor in cardiovascular disease," Circulation, vol. 117, no. 12, pp. 1594-1602, 2008.

[9] C. Rammos, U. B. Hendgen-Cotta, J. Sobierajski et al., "Macrophage migration inhibitory factor is associated with vascular dysfunction in patients with end-stage renal disease," International Journal of Cardiology, vol. 168, no. 6, pp. 52495256, 2013.

[10] J. L. Fleg, W. S. Aronow, and W. H. Frishman, "Cardiovascular drug therapy in the elderly: benefits and challenges," Nature Reviews Cardiology, vol. 8, no. 1, pp. 13-28, 2011.

[11] I. B. Jeffery and P. W. O'Toole, "Diet-microbiota interactions and their implications for healthy living," Nutrients, vol. 5, no. 1, pp. 234-252, 2013.

[12] F. Sofi, F. Cesari, R. Abbate, G. F. Gensini, and A. Casini, "Adherence to Mediterranean diet and health status: metaanalysis," British Medical Journal, vol. 337, article al344, 2008.

[13] F. J. Larsen, B. Ekblom, K. Sahlin, J. O. Lundberg, and E. Weitzberg, "Effects of dietary nitrate on blood pressure in healthy volunteers," The New England Journal of Medicine, vol. 355, no. 26, pp. 2792-2793, 2006.

[14] J. O. Lundberg, E. Weitzberg, and M. T. Gladwin, “The nitratenitrite-nitric oxide pathway in physiology and therapeutics," Nature Reviews Drug Discovery, vol. 7, no. 2, pp. 156-167, 2008.

[15] M. Totzeck, U. B. Hendgen-Cotta, P. Luedike et al., "Nitrite regulates hypoxic vasodilation via myoglobin-dependent nitric oxide generation," Circulation, vol. 126, no. 3, pp. 325-334, 2012.

[16] M. T. Gladwin, A. N. Schechter, D. B. Kim-Shapiro et al., "The emerging biology of the nitrite anion," Nature Chemical Biology, vol. 1, no. 6, pp. 308-314, 2005.

[17] T. Rassaf, L. W. Poll, P. Brouzos et al., "Positive effects of nitric oxide on left ventricular function in humans," European Heart Journal, vol. 27, no. 14, pp. 1699-1705, 2006.

[18] C. Rammos, U. B. Hendgen-Cotta, J. Sobierajski, A. Bernard, M. Kelm, and T. Rassaf, "Dietary nitrate reverses vascular dysfunction in older adults with moderately increased cardiovascular risk," Journal of the American College of Cardiology, vol. 63, no. 15, pp. 1584-1585, 2014.

[19] J. Sobierajski, U. B. Hendgen-Cotta, P. Luedike et al., "Assessment of macrophage migration inhibitory factor in humans: protocol for accurate and reproducible levels," Free Radical Biology and Medicine, vol. 63, pp. 236-242, 2013.

[20] A. L. Pauca, M. F. O’Rourke, and N. D. Kon, "Prospective evaluation of a method for estimating ascending aortic pressure from the radial artery pressure waveform," Hypertension, vol. 38, no. 4, pp. 932-937, 2001.

[21] S. G. Wannamethee, P. Welsh, G. D. Lowe et al., "N-terminal pro-brain natriuretic peptide is a more useful predictor of cardiovascular disease risk than C-reactive protein in older men with and without pre-existing cardiovascular disease," Journal of the American College of Cardiology, vol. 58, no. 1, pp. 56-64, 2011. 
[22] S. Tsimikas, P. Willeit, J. Willeit et al., "Oxidation-specific biomarkers, prospective 15-year cardiovascular and stroke outcomes, and net reclassification of cardiovascular events," Journal of the American College of Cardiology, vol. 60, no. 21, pp. 22182229, 2012.

[23] H. Sell, C. Habich, and J. Eckel, "Adaptive immunity in obesity and insulin resistance," Nature Reviews Endocrinology, vol. 8, no. 12, pp. 709-716, 2012.

[24] P. M. Ridker, M. Cushman, M. J. Stampfer, R. P. Tracy, and C. H. Hennekens, "Inflammation, aspirin, and the risk of cardiovascular disease in apparently healthy men," The New England Journal of Medicine, vol. 336, no. 14, pp. 973-979, 1997.

[25] K. Iseki, M. Tozawa, S. Yoshi, and K. Fukiyama, "Serum Creactive protein (CRP) and risk of death in chronic dialysis patients," Nephrology Dialysis Transplantation, vol. 14, no. 8, pp. 1956-1960, 1999.

[26] A. Schober, J. Bernhagen, and C. Weber, "Chemokine-like functions of MIF in atherosclerosis," Journal of Molecular Medicine, vol. 86, no. 7, pp. 761-770, 2008.

[27] Y. Asare, M. Schmitt, and J. Bernhagen, "The vascular biology of macrophage migration inhibitory factor (MIF): expression and effects in inflammation, atherogenesis and angiogenesis," Thrombosis and Haemostasis, vol. 109, no. 3, pp. 391-398, 2013.

[28] L. Chen, G. Yang, X. Zhang et al., "Induction of MIF expression by oxidized LDL via activation of NF- $\kappa$ B in vascular smooth muscle cells," Atherosclerosis, vol. 207, no. 2, pp. 428-433, 2009.

[29] C. Rammos, U. B. Hendgen-Cotta, R. Deenen et al., "Agerelated vascular gene expression profiling in mice," Mechanisms of Ageing and Development, vol. 135, pp. 15-23, 2014.

[30] P. M. Ridker, M. J. Stampfer, and N. Rifai, "Novel risk factors for systemic atherosclerosis: a comparison of C-reactive protein, fibrinogen, homocysteine, lipoprotein(a), and standard cholesterol screening as predictors of peripheral arterial disease," The Journal of the American Medical Association, vol. 285, no. 19, pp. 2481-2485, 2001.

[31] P. J. Barter, S. Nicholls, K. Rye, G. M. Anantharamaiah, M. Navab, and A. M. Fogelman, "Antiinflammatory properties of HDL," Circulation Research, vol. 95, no. 8, pp. 764-772, 2004.

[32] A. Burger-Kentischer, H. Göbel, R. Kleemann et al., "Reduction of the aortic inflammatory response in spontaneous atherosclerosis by blockade of macrophage migration inhibitory factor (MIF)," Atherosclerosis, vol. 184, no. 1, pp. 28-38, 2006.

[33] J. M. Harper, J. E. Wilkinson, and R. A. Miller, "Macrophage migration inhibitory factor-knockout mice are long lived and respond to caloric restriction," The FASEB Journal, vol. 24, no. 7, pp. 2436-2442, 2010.

[34] L. J. Appel, T. J. Moore, E. Obarzanek et al., "A clinical trial of the effects of dietary patterns on blood pressure. DASH Collaborative Research Group," The New England Journal of Medicine, vol. 336, no. 16, pp. 1117-1124, 1997.

[35] L. J. Appel, F. M. Sacks, V. J. Carey et al., "Effects of protein, monounsaturated fat, and carbohydrate intake on blood pressure and serum lipids: results of the OmniHeart randomized trial," Journal of the American Medical Association, vol. 294, no. 19, pp. 2455-2464, 2005.

[36] H. Schroeter, C. Heiss, J. Balzer et al., “(-)-Epicatechin mediates beneficial effects of flavanol-rich cocoa on vascular function in humans," Proceedings of the National Academy of Sciences of the United States of America, vol. 103, no. 4, pp. 1024-1029, 2006.

[37] U. B. Hendgen-Cotta, P. Luedike, M. Totzeck et al., "Dietary nitrate supplementation improves revascularization in chronic ischemia," Circulation, vol. 126, no. 16, pp. 1983-1992, 2012.
[38] U. B. Hendgen-Cotta, M. Kelm, and T. Rassaf, "A highlight of myoglobin diversity: the nitrite reductase activity during myocardial ischemia-reperfusion," Nitric Oxide-Biology and Chemistry, vol. 22, no. 2, pp. 75-82, 2010.

[39] T. Rassaf, M. Totzeck, U. B. Hendgen-Cotta, S. Shiva, G. Heusch, and M. Kelm, "Circulating nitrite contributes to cardioprotection by remote ischemic preconditioning," Circulation Research, vol. 114, no. 10, pp. 1601-1610, 2014.

[40] A. L. Sindler, A. E. Devan, B. S. Fleenor, and D. R. Seals, "Inorganic nitrite supplementation for healthy arterial aging," Journal of Applied Physiology, vol. 116, no. 5, pp. 463-477, 2014.

[41] M. Bahra, V. Kapil, V. Pearl, S. Ghosh, and A. Ahluwalia, "Inorganic nitrate ingestion improves vascular compliance but does not alter flow-mediated dilatation in healthy volunteers," Nitric Oxide: Biology and Chemistry, vol. 26, no. 4, pp. 197-202, 2012.

[42] J. Yeboah, J. R. Crouse, F. Hsu, G. L. Burke, and D. M. Herrington, "Brachial flow-mediated dilation predicts incident cardiovascular events in older adults: the cardiovascular health study," Circulation, vol. 115, no. 18, pp. 2390-2397, 2007.

[43] T. W. Hansen, J. A. Staessen, C. Torp-Pedersen et al., "Prognostic value of aortic pulse wave velocity as index of arterial stiffness in the general population," Circulation, vol. 113, no. 5, pp. 664-670, 2006.

[44] P. Luedike, U. B. Hendgen-Cotta, J. Sobierajski et al., "Cardioprotection through S-nitros(yl)ation of macrophage migration inhibitory factor," Circulation, vol. 125, no. 15, pp. 1880-1889, 2012.

[45] T. Rassaf, C. Weber, and J. Bernhagen, "Macrophage migration inhibitory factor in myocardial ischemia/reperfusion injury," Cardiovascular Research, vol. 102, no. 2, pp. 321-328, 2014.

[46] A. Santoro, E. Pini, M. Scurti et al., "Combating inflammaging through a Mediterranean whole diet approach: the NU-AGE project's conceptual framework and design," Mechanisms of Ageing and Development, vol. 136-137, pp. 3-13, 2014.

[47] A. L. Sindler, B. S. Fleenor, J. W. Calvert et al., "Nitrite supplementation reverses vascular endothelial dysfunction and large elastic artery stiffness with aging," Aging Cell, vol. 10, no. 3, pp. 429-437, 2011.

[48] M. J. Roman, R. B. Devereux, J. E. Schwartz et al., "Arterial stiffness in chronic inflammatory diseases," Hypertension, vol. 46, no. 1, pp. 194-199, 2005.

[49] S. Laurent, J. Cockcroft, L. van Bortel et al., "Expert consensus document on arterial stiffness: methodological issues and clinical applications," European Heart Journal, vol. 27, no. 21, pp. 2588-2605, 2006.

[50] C. M. McEniery, I. R. Hall, A. Qasem, I. B. Wilkinson, and J. R. Cockcroft, "Normal vascular aging: differential effects on wave reflection and aortic pulse wave velocity. The Anglo-Cardiff Collaborative Trial (ACCT)," Journal of the American College of Cardiology, vol. 46, no. 9, pp. 1753-1760, 2005.

[51] J. J. Oliver and D. J. Webb, "Noninvasive assessment of arterial stiffness and risk of atherosclerotic events," Arteriosclerosis, Thrombosis, and Vascular Biology, vol. 23, no. 4, pp. 554-566, 2003.

[52] G. Mancia, G. de Backer, A. Dominiczak et al., "2007 Guidelines for the management of arterial hypertension. The Task Force for the Management of Arterial Hypertension of the European Society of Hypertension (ESH) and of the European Society of Cardiology (ESC)," European Heart Journal, vol. 28, no. 12, pp. 1462-1536, 2007. 
[53] V. Kapil, A. B. Milsom, M. Okorie et al., "Inorganic nitrate supplementation lowers blood pressure in humans: role for nitrite-derived No," Hypertension, vol. 56, no. 2, pp. 274-281, 2010.

[54] M. Pirro, G. Schillaci, G. Savarese et al., "Attenuation of inflammation with short-term dietary intervention is associated with a reduction of arterial stiffness in subjects with hypercholesterolaemia," The European Journal of Cardiovascular Prevention and Rehabilitation, vol. 11, no. 6, pp. 497-502, 2004. 


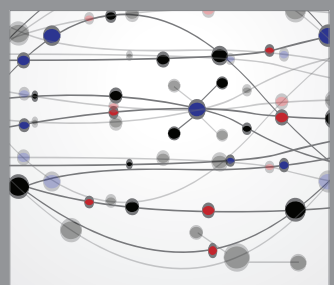

The Scientific World Journal
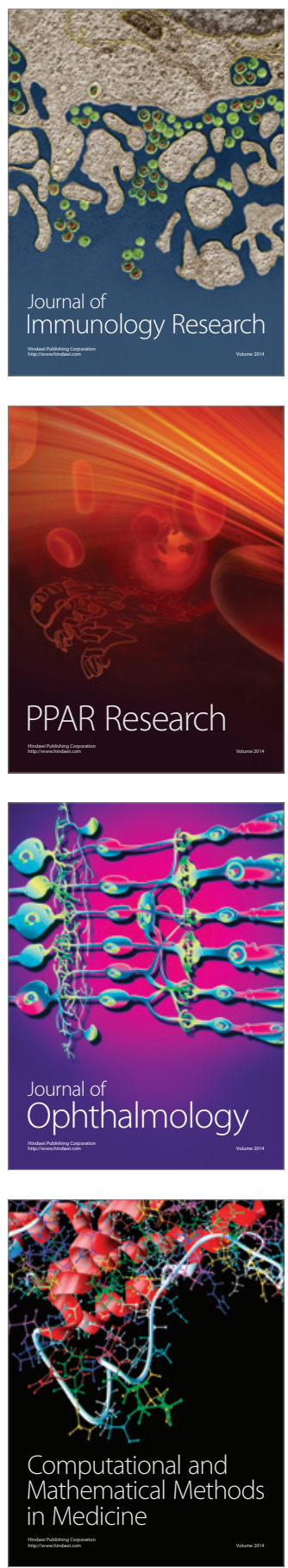

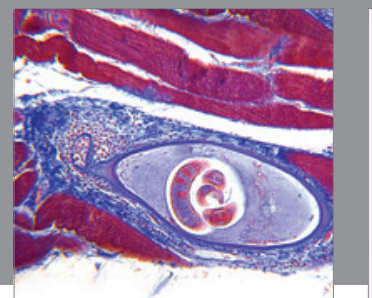

Gastroenterology

Research and Practice
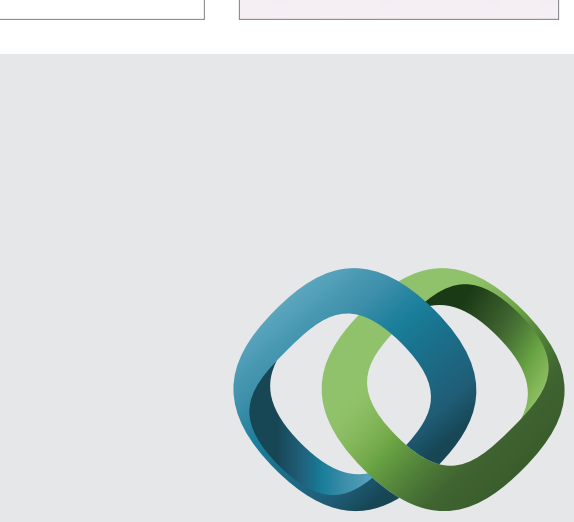

\section{Hindawi}

Submit your manuscripts at

http://www.hindawi.com
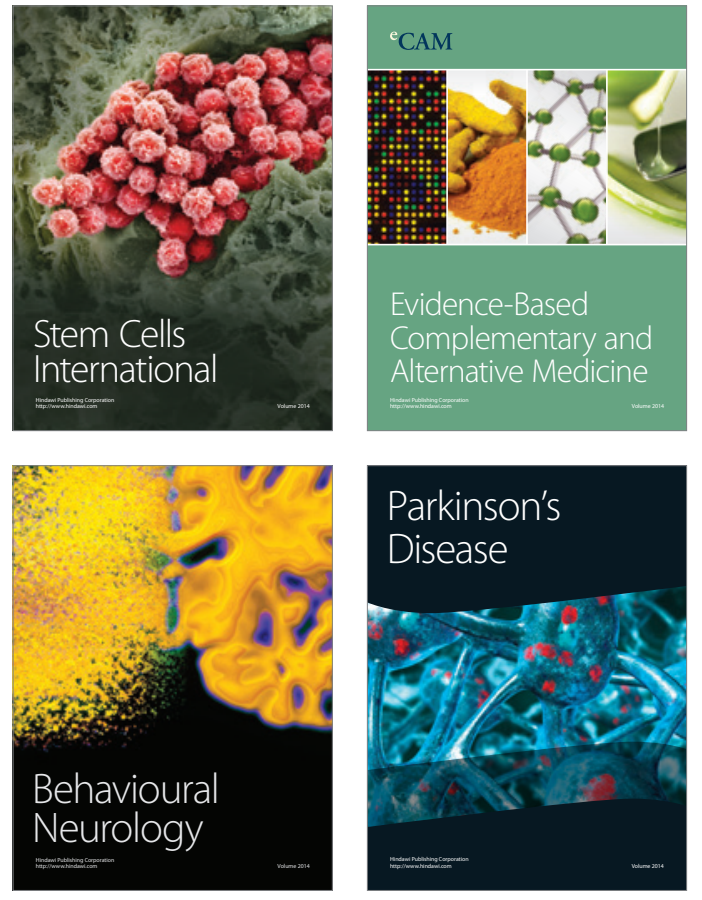
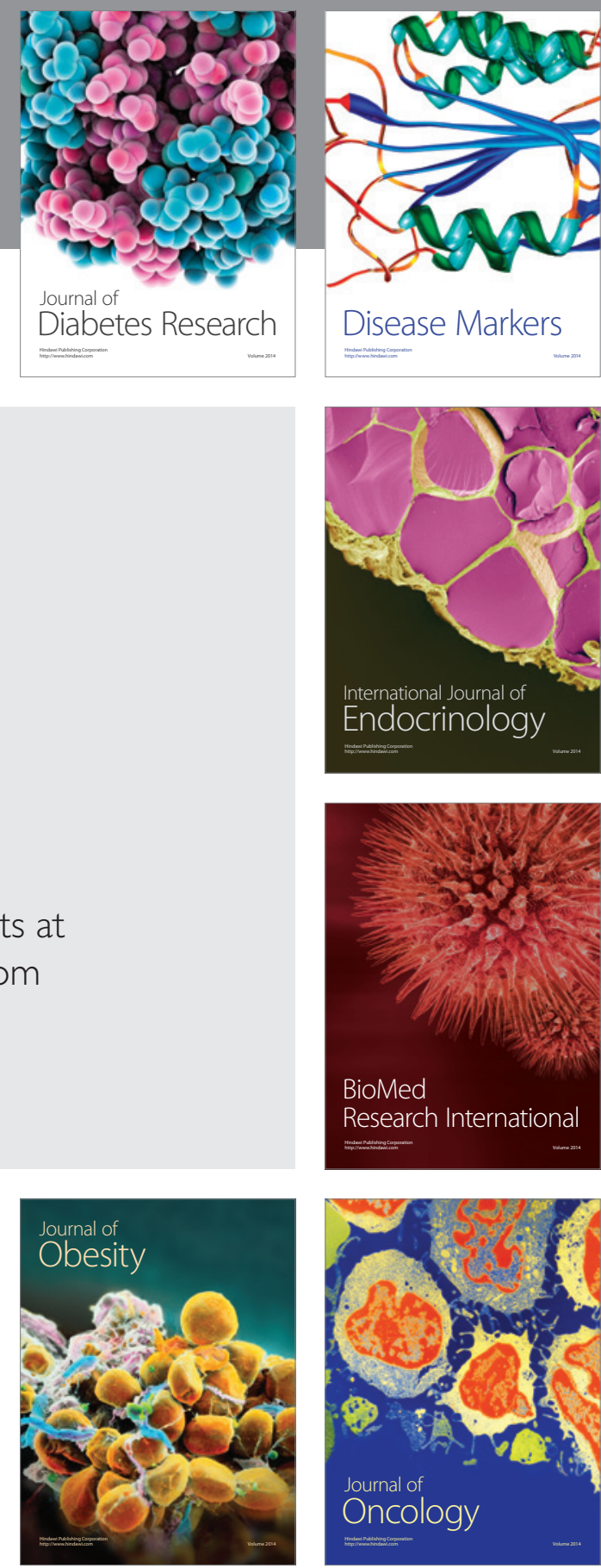

Disease Markers
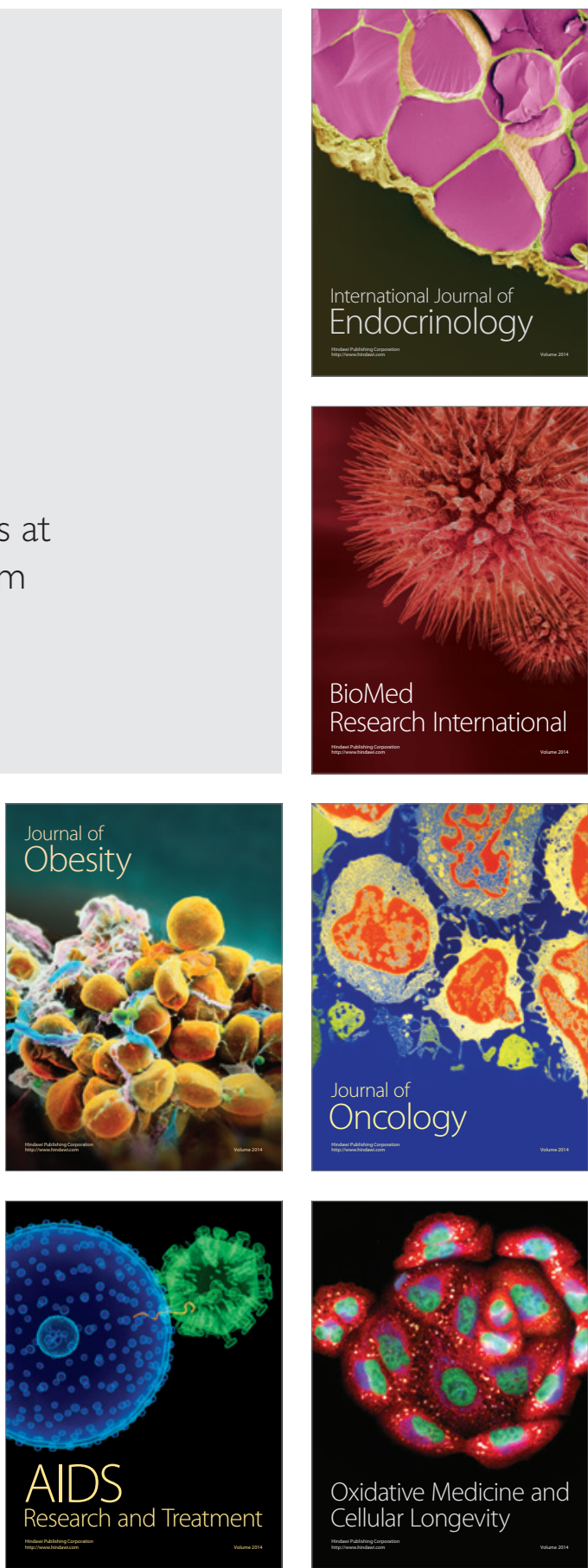